\title{
Spinopelvic Alignment and Low Back Pain after Total Hip Replacement Arthroplasty in Patients with Severe Hip Osteoarthritis
}

\author{
Yawara Eguchi ${ }^{1}$, Satoshi Iida ${ }^{2}$, Chiho Suzuki ${ }^{2}$, Yoshiyuki Shinada ${ }^{2}$, \\ Tomoko Shoji ${ }^{2}$, Kazuhisa Takahashi ${ }^{3}$, Seiji Ohtori ${ }^{3}$ \\ ${ }^{1}$ Department of Orthopedic Surgery, Shimoshizu National Hospital, Yotsukaido, Japan \\ ${ }^{2}$ Department of Orthopedic Surgery, Matsudo City Hospital, Matsudo, Japan \\ ${ }^{3}$ Department of Orthopaedic Surgery, Graduate School of Medicine, Chiba University, Chiba, Japan
}

Study Design: Retrospective observational study.

Purpose: We examined change in lumbrosacral spine alignment and low back pain (LBP) following total hip arthroplasty (THA) in patients with severe hip osteoarthritis (OA).

Overview of Literature: Severe hip osteoarthritis has been reported to cause spine alignment abnormalities and low back pain, and it has been reported that low back pain is improved following THA.

Methods: Our target population included 30 patients (29 female, mean age 63.5 years) with hip 0A who underwent direct anterior approach THA. There were 12 cases with bilateral hip disease and 18 cases with unilateral osteoarthritis. Visual analogue scale (VAS) scores for LBP and coxalgia, the Roland-Morris Disability Questionnaire (RDQ), and the Japanese Orthopaedic Association Back Pain Evaluation Questionnaire (JOABPE0) were assessed before and after surgery. Spinal alignment metrics were measured before and after surgery.

Results: VAS for LBP change from preoperative to final postoperative observation was significantly improved ( $p<0.05$ ), as was VAS for hip pain ( $p<0.001)$. RDQ improved significantly $(p<0.01)$. All five domains of JOABPEQ were significantly improved $(p<0.05)$. In terms of coronal alignment, lumbar scoliosis change from preoperative to last observation was significantly reduced $(p<0.05)$. There were no significant changes in the sagittal alignment metrics. In addition, there was a correlation between before and after RDQ difference and before and after lumbar scoliosis difference $(p<0.05)$. VAS for LBP $(p<0.05)$ as well as RDQ $(p<0.05)$ were significantly improved only in unilateral $\mathrm{OA}$. Lumbar scoliosis was significantly improved in cases of unilateral $\mathrm{OA}(p<0.05)$, but alignment did not improve in cases of bilateral $O A(p=0.29)$.

Conclusions: The present study demonstrates improvements in VAS for LBP, RDQ, and all domains of JOABPEO. There were also significant reductions in lumbar scoliosis and an observed correlation of RDQ improvement with lumbar scoliosis improvement. We were able to observe improvements in lumbar scoliosis and low back pain only in cases of unilateral OA. It has been suggested that the mechanism of low back pain improvement following THA is related to compensatory lumbar scoliosis improvement.

Keywords: Hip osteoarthritis; Total hip replacement arthroplasty; Low back pain

Received Apr 26, 2017; Revised Aug 6, 2017; Accepted Aug 21, 2017

Corresponding author: Yawara Eguchi

Department of Orthopedic Surgery, Shimoshizu National Hospital, 934-5, Shikawatashi, Yotsukaido, Chiba, 284-0003, Japan

Tel: +81-43-422-2511, Fax: +81-43-421-3007, E-mail: yawara_eguchi@yahoo.co.jp 


\section{Introduction}

Severe hip osteoarthritis (OA) may cause abnormal spinal alignment and may also be associated with low back pain (LBP). This syndrome (hip-spine syndrome) was originally described by Offierski and MacNab [1] in 1983. LBP and spinal function have been reported to improve following total hip arthroplasty (THA) [2,3]. However, the precise cause of such an improvement of LBP following THA remains unknown.

The Japanese Orthopedic Association Back Pain Evaluation Questionnaire (JOABPEQ) [4] is based on the Roland-Morris Disability Questionnaire (RDQ) [5] and Short Form 36. JOABPEQ provides specific multidimensional outcome measures for patients with LBP, including metrics of dysfunction and disability caused by the disease and resulting psychosocial problems.

No previous study has assessed the association between spinopelvic alignment, including coronal alignment and LBP, using patient-based questionnaire data following THA. The purpose of this study was to evaluate the effect of THA on spinopelvic alignment and LBP using JOABPEQ and RDQ in patients with severe hip OA.

\section{Materials and Methods}

All subjects provided written informed consent prior to participating in the study, which was approved by the Institutional Review Board of Matsudo City Hospital (IRB approval no., No28-5). We declare that this research involving human subjects have been performed in accordance with the ethical standards laid down in the 1964 Declaration of Helsinki and its later amendments.

There were 50 patients with OA of the hip who underwent direct anterior approach THA between June 2011 and November 2011 at Matsudo City Hospital. After excluding patients with a history of treatment for LBP related to spinal disorders, 30 patients (one male and 29 females; mean age, 63.5 years; range, $45-78$ years) were included in our analysis. In total, 19 patients underwent surgery of the right hip, and 11 underwent surgery of the left hip. There were 12 patients with bilateral OA (one male, 11 females; mean age, 62.2 years; range, 45-78 years) and 18 patients with unilateral OA (18 females; mean age, 64.4 years; range, $51-77$ years). Of the 12 patients with bilateral OA, six developed OA following contralateral THA.
The exclusion criteria were as follows: (1) previous lumbar spinal surgery; (2) lumbar canal stenosis; (3) lumbar disc herniation; (4) myelopathy; and (5) spinal tumor, infectious disease, or spinal trauma.

LBP assessments and lumbosacral spinal alignment measurements from standing radiographs were performed preoperatively, 1 month postoperatively, and periodically until the final observation (mean, 126.9 days).

Clinical symptoms were evaluated using the Visual Analogue Scale (VAS) scores for LBP and coxalgia ranging from $100 \mathrm{~mm}$ (extreme amount of pain) to $0 \mathrm{~mm}$ (no pain), the Japanese Orthopedic Association (JOA, 0-29 points) scoring system (Table 1), and the RDQ (0-24 points) [5]. The normal JOA score is 29 points, which is derived on the basis of three subjective symptoms ( 9 points), three clinical signs including straight-leg raise (6 points), and seven activities of daily living (ADL, 14 points). The normal RDQ is 0 points with the total number of items checked from a minimum of 0 to a maximum of 24.

JOABPEQ includes 25 questions (Table 2), and scores are calculated on the basis of the answers to these questions in the following five domains: pain-related disorders, lumbar spine dysfunction, gait disturbance, social life dysfunction, and psychological disorders. Scores for each domain are calculated according to the official guidelines and range from 0 to 100 points, which are deemed proportional to the patient's clinical condition [4].

The frontal view of the entire spine and the lateral view that includes the hip joints were photographed with the patient in the standing position [6]. Radiographic measurements were of lumbar scoliosis (LS), sacral slope (SS), lumbar lordosis (LL), pelvic tilt (PT), and pelvic incidence (PI) were performed before and after surgery. LS was measured as the angle between the lower endplate of L1 and the lower endplate of L5 on frontal radiographs. LL was measured from the lower endplate of T12 to the upper endplate of S1. PT was measured as the angle between the vertical line and the line joining the hip axis to the center of the superior endplate of S1. PI was measured as the angle subtended by a perpendicular line from the upper endplate of S1 and a line connecting the center of the femoral head to the center of the cephalad endplate of S1. SS was measured as the angle between the superior endplate of $\mathrm{S} 1$ and a horizontal line.

Statistical analyses were performed using StatView software ver. 5.0 (SAS Institute Inc., Cary, NC, USA). 

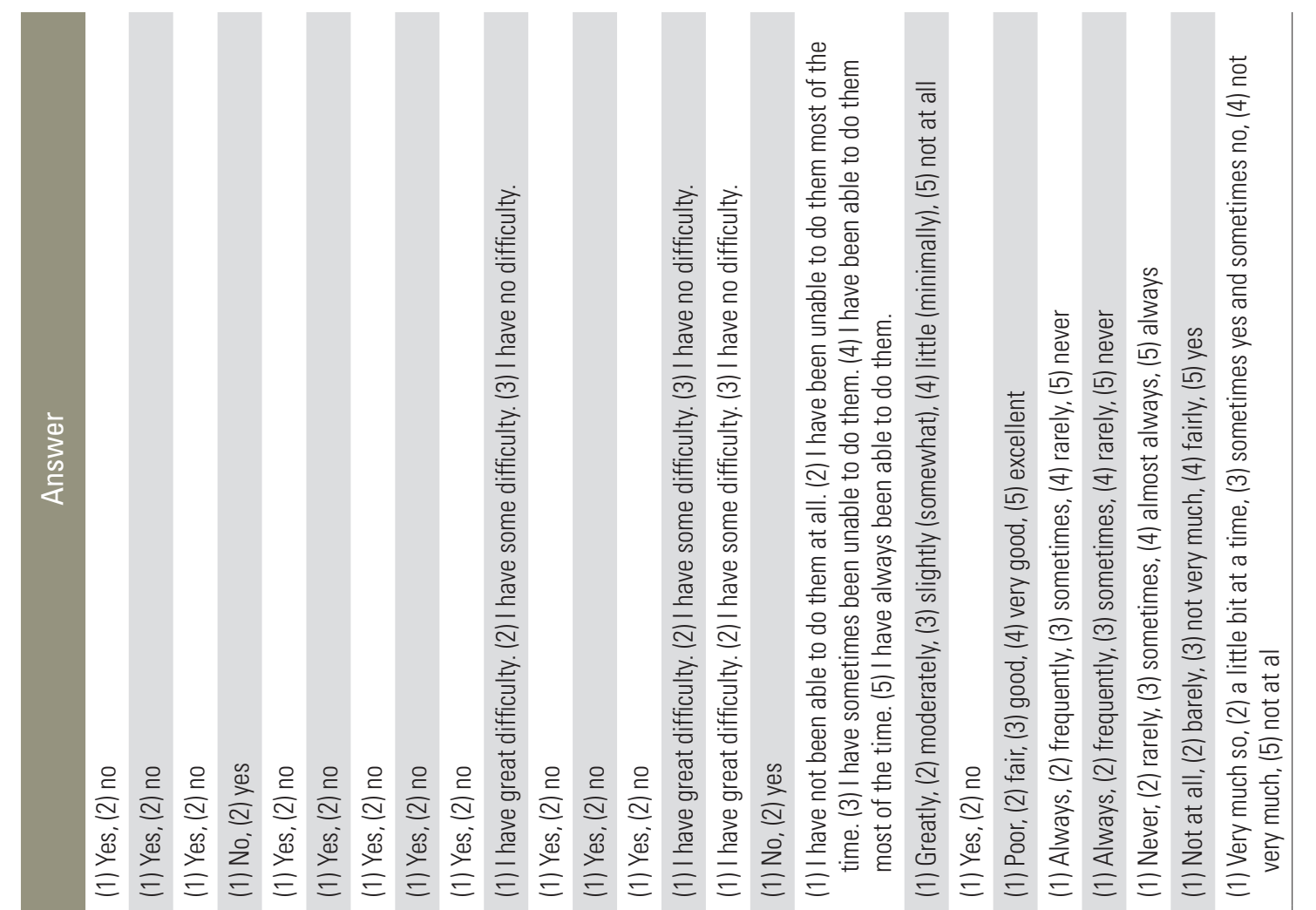

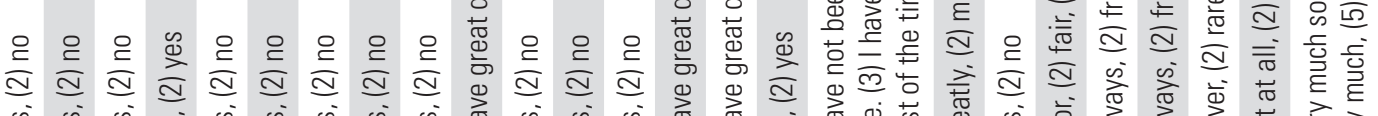

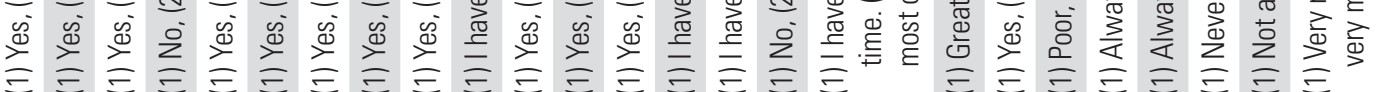

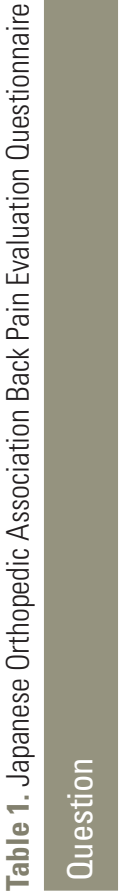

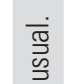
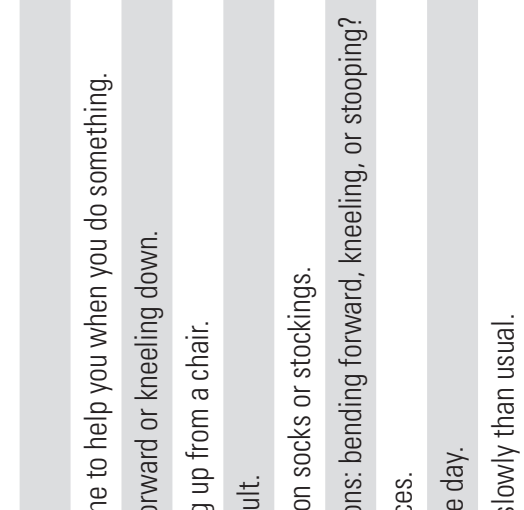
Differences between LBP assessments before and after surgery and lumbosacral spine alignment were evaluated using unpaired $t$-test. A threshold of $p<0.05$ was consid- ered significant. Results are presented as mean \pm standard error.

Table 2. Radiographic parameters and clinical symptoms of total patients

\begin{tabular}{|c|c|c|c|}
\hline Variable & Preoperative & Final & $p$-value \\
\hline \multicolumn{4}{|l|}{ Radiographic parameters } \\
\hline Lumbar scoliosis $\left({ }^{\circ}\right)$ & $6.557 \pm 0.834$ & $3.969 \pm 0.677$ & 0.019 \\
\hline Sacral slope $\left({ }^{\circ}\right)$ & $35.57 \pm 1.88$ & $33.21 \pm 1.76$ & 0.365 \\
\hline Lumbar lordosis $\left({ }^{\circ}\right)$ & $45.81 \pm 2.95$ & $43.70 \pm 2.83$ & 0.608 \\
\hline Pelvic tilt $\left({ }^{\circ}\right)$ & $12.70 \pm 1.19$ & $12.92 \pm 1.38$ & 0.903 \\
\hline Pelvic incidence $\left({ }^{\circ}\right)$ & $51.48 \pm 1.87$ & $49.14 \pm 1.85$ & 0.379 \\
\hline \multicolumn{4}{|l|}{ Clinical symptoms } \\
\hline VAS (low back pain) (mm) & $22.79 \pm 4.69$ & $9.35 \pm 3.30$ & 0.023 \\
\hline VAS (coxalgia) (mm) & $63.72 \pm 4.63$ & $3.89 \pm 2.50$ & 0.00012 \\
\hline Roland-Morris Disability Questionnaire & $5.30 \pm 0.98$ & $1.47 \pm 0.66$ & 0.002 \\
\hline \multicolumn{4}{|c|}{ Japanese Orthopedic Association Back Pain Evaluation Questionnaire } \\
\hline Pain-related disorders & $81.96 \pm 4.81$ & $94.48 \pm 3.83$ & 0.0149 \\
\hline Lumbar spine dysfunction & $66.17 \pm 5.05$ & $84.10 \pm 3.62$ & 0.0166 \\
\hline Gait disturbance & $52.03 \pm 4.36$ & $83.03 \pm 4.08$ & 0.00065 \\
\hline Social life dysfunction & $52.0 \pm 2.90$ & $78.92 \pm 3.35$ & 0.0162 \\
\hline Psychological disorders & $57.32 \pm 2.19$ & $72.89 \pm 3.07$ & 0.00013 \\
\hline
\end{tabular}

Values are presented as mean \pm standard error. Bold type is considered statistically significant $(p<0.05)$.

VAS, Visual Analogue Scale.
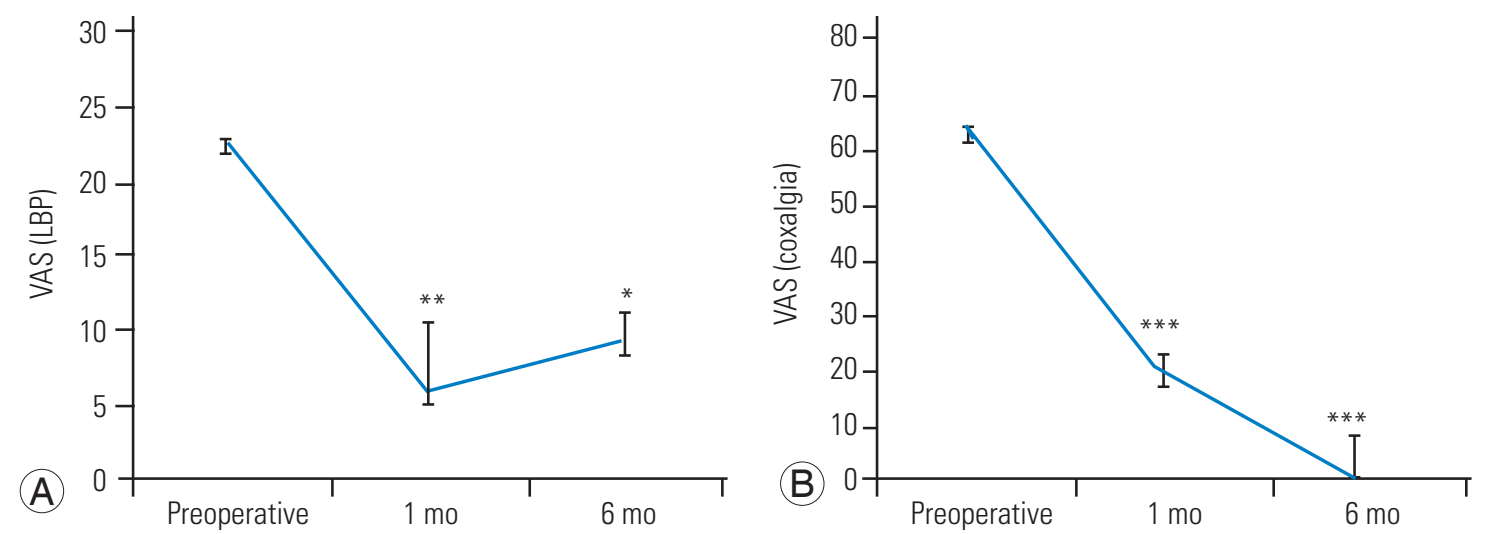

Fig. 1. VAS scores for LBP (A) and coxalgia (B) in patients before and after total hip arthroplasty. Preoperative, 1-month postoperative, and final observation low back pain VAS scores significantly improved. These were $22.79 \mathrm{~mm} \rightarrow 6.0 \mathrm{~mm}(p=0.0019)$ $\rightarrow 9.35 \mathrm{~mm}(p=0.023)$, respectively; similarly for hip pain, VAS scores were $63.72 \mathrm{~mm} \rightarrow 20.32 \mathrm{~mm}(p=0.00033) \rightarrow 3.89 \mathrm{~mm}$ $(p=0.00012)$, respectively. VAS, Visual Analogue Scale; LBP, low back pain. ${ }^{*} p<0.05,{ }^{* *} p<0.01,{ }^{* * *} p<0.001$. 


\section{Results}

The final observation period was $126.9 \pm 55.5$ days, operation time was $108.9 \pm 4.9$ minutes, and blood loss was 693.8 $\pm 79.2 \mathrm{~mL}$. There were no complications during the surgeries.

Preoperative, 1-month postoperative, and final observation LBP VAS scores were $22.79 \pm 4.69 \mathrm{~mm}, 6.0 \pm 1.91$ $\mathrm{mm}(p=0.0019), 9.35 \pm 3.30 \mathrm{~mm}(p=0.023)$, respectively. Preoperative, 1-month postoperative, and final observation scores for hip pain VAS were $63.72 \pm 4.63$ $\mathrm{mm}, 20.32 \pm 5.01 \mathrm{~mm}(p=0.00033)$, and $3.89 \pm 2.50 \mathrm{~mm}$ $(p=0.00012)$, respectively, which showed significant improvements (Fig. 1). RDQ showed significant improvement from preoperative $(5.30 \pm 0.98)$ to final observation

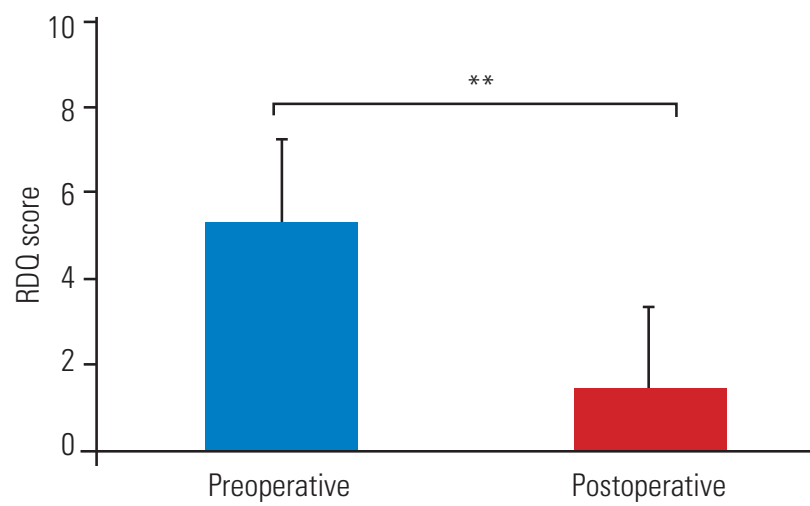

Fig. 2. RDQ scores of patients before and after total hip arthroplasty. Final observation RDQ scores significantly improved from preoperative 5.30 to 1.47 points ( $p=0.002$ ). RDQ, Roland-Morris Disability Questionnaire. ${ }^{* *} p<0.01$.
$(1.47 \pm 0.66)(p=0.002)$ (Fig. 2, Table 2). Each respective JOABPEQ domain showed significant improvement (preoperative-to-final observation, respectively, all) as follows: pain-related disorders, $81.96 \pm 4.81$ to $94.48 \pm 3.83$ ( $p=0.0149$ ); lumbar spine dysfunction, $66.17 \pm 5.05$ to $84.10 \pm 3.62(p=0.0166)$; gait disturbance, $52.03 \pm 4.36$ to $83.03 \pm 4.08(p=0.00065)$; social life disturbance, $52.0 \pm 2.90$ to $78.92 \pm 3.35(p=0.0162)$; and psychological disorders, $57.32 \pm 2.19$ to $72.89 \pm 3.07(p=0.00013$ ) (Fig. 3, Table 2).

Regarding spinal alignment metrics, LS significantly reduced from preoperative $\left(6.557^{\circ} \pm 0.834^{\circ}\right)$ to final observation $\left(3.969^{\circ} \pm 0.677^{\circ}\right)(p=0.0194)$ (Fig. 4, Table $2)$. In contrast, there were no significant differences in the other metrics (preoperative-to-final observation, respectively, all) of SS, $35.57^{\circ} \pm 1.88^{\circ}$ to $33.21^{\circ} \pm 1.76^{\circ}$, $p=0.364$; LL, $45.81^{\circ} \pm 2.95^{\circ}$ to $43.70^{\circ} \pm 2.83^{\circ}, p=0.608$; $\mathrm{PT}, 12.70^{\circ} \pm 1.19^{\circ}$ to $12.92^{\circ} \pm 1.38^{\circ}, p=0.903$; or $\mathrm{PI}$, $51.48^{\circ} \pm 1.87^{\circ}$ to $49.14^{\circ} \pm 1.855^{\circ}, p=0.378$ (Fig. 4 , Table 2). The preoperative-to-postoperative RDQ difference and the preoperative-to-postoperative LS difference were correlated ( $r=0.437, p=0.015$ ) (Fig. 5).

Regarding the comparison of radiographic parameters between unilateral and bilateral OA, LS significantly reduced from preoperative $\left(7.21^{\circ} \pm 1.14^{\circ}\right)$ to final observation $\left(4.12^{\circ} \pm 0.85^{\circ}\right)(p=0.037)$ in unilateral OA. There were no significant differences in LS between preoperative $\left(5.50^{\circ} \pm 1.16^{\circ}\right)$ to final observation $\left(3.73^{\circ} \pm 1.16^{\circ}\right)(p=0.29)$ in bilateral OA. There were no significant differences in any other radiographic parameter for either group (Table 3).

A comparison of the clinical symptoms between

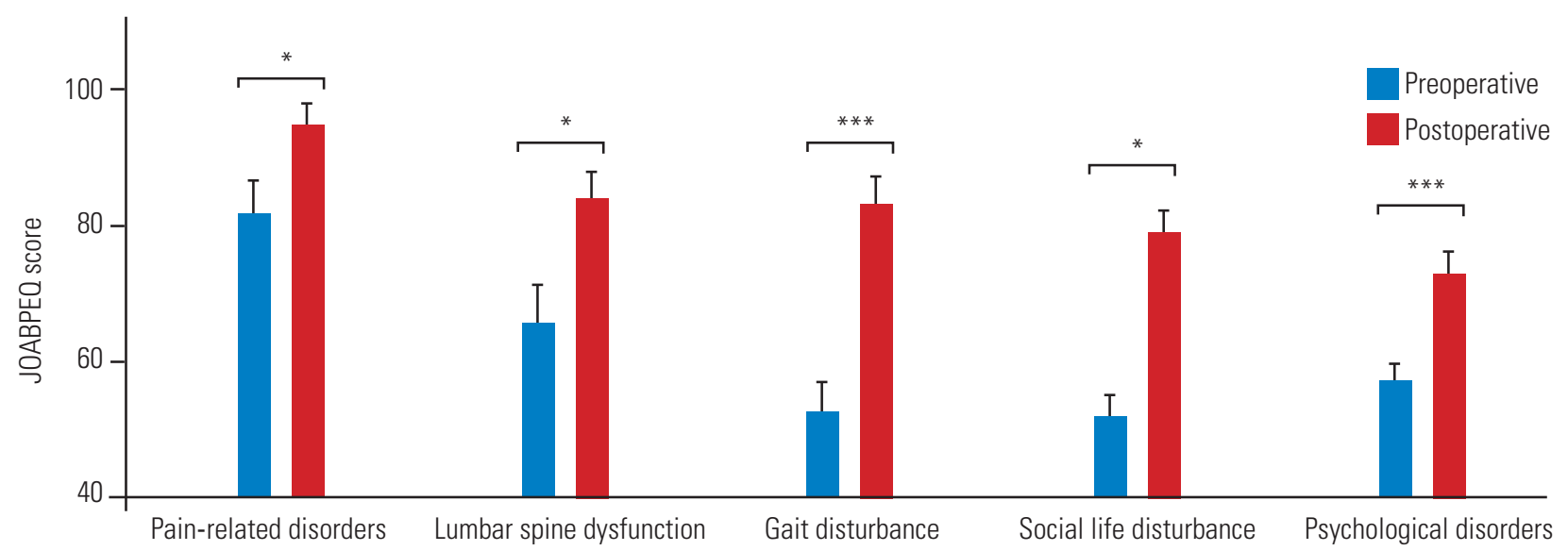

Fig. 3. JOABPEO scores of patients before and after total hip arthroplasty. All JOABPEO domains significantly improved (from preoperative-to-final observation, respectively, all): pain-related disorders (from 81.96 to 94.48, $p=0.0149$ ); lumbar spine dysfunction (from 66.17 to $84.10, p=0.0166$ ); gait disturbance (from 52.03 to 83.03, $p=0.00065$ ); social life disturbance (from 52.0 to 78.92, $p=0.0162$ ); psychological disorders (from 57.32 to 72.89 , $p=0.00013)$. JOABPEQ, Japanese Orthopedic Association Back Pain Evaluation Questionnaire. ${ }^{*} p<0.05,{ }^{* * *} p<0.001$. 
unilateral and bilateral $\mathrm{OA}$ revealed that in unilateral OA, LBP VAS $(21.18 \pm 6.15 \mathrm{~mm}$ to $4.76 \pm 1.93 \mathrm{~mm}$, $p=0.016)$, hip pain VAS $(59.65 \pm 6.31 \mathrm{~mm}$ to $2.12 \pm 0.75$ $\mathrm{mm}, p=0.0000001)$, and RDQ $(4.72 \pm 1.07$ to $0.83 \pm 0.52$, $p=0.002$ ) significantly improved from preoperative-to- final observation (Table 3). In bilateral OA, although hip pain VAS significantly improved $(69.50 \pm 6.69 \mathrm{~mm}$ to $6.64 \pm 6.34 \mathrm{~mm}, p=0.000001)$, there were no significant improvements in LBP VAS $(25.27 \pm 7.55 \mathrm{~mm}$ to $8.10 \pm 4.08$ $\mathrm{mm}, p=0.07)$ and RDQ $(6.17 \pm 1.90$ to $2.42 \pm 1.44, p=0.13)$
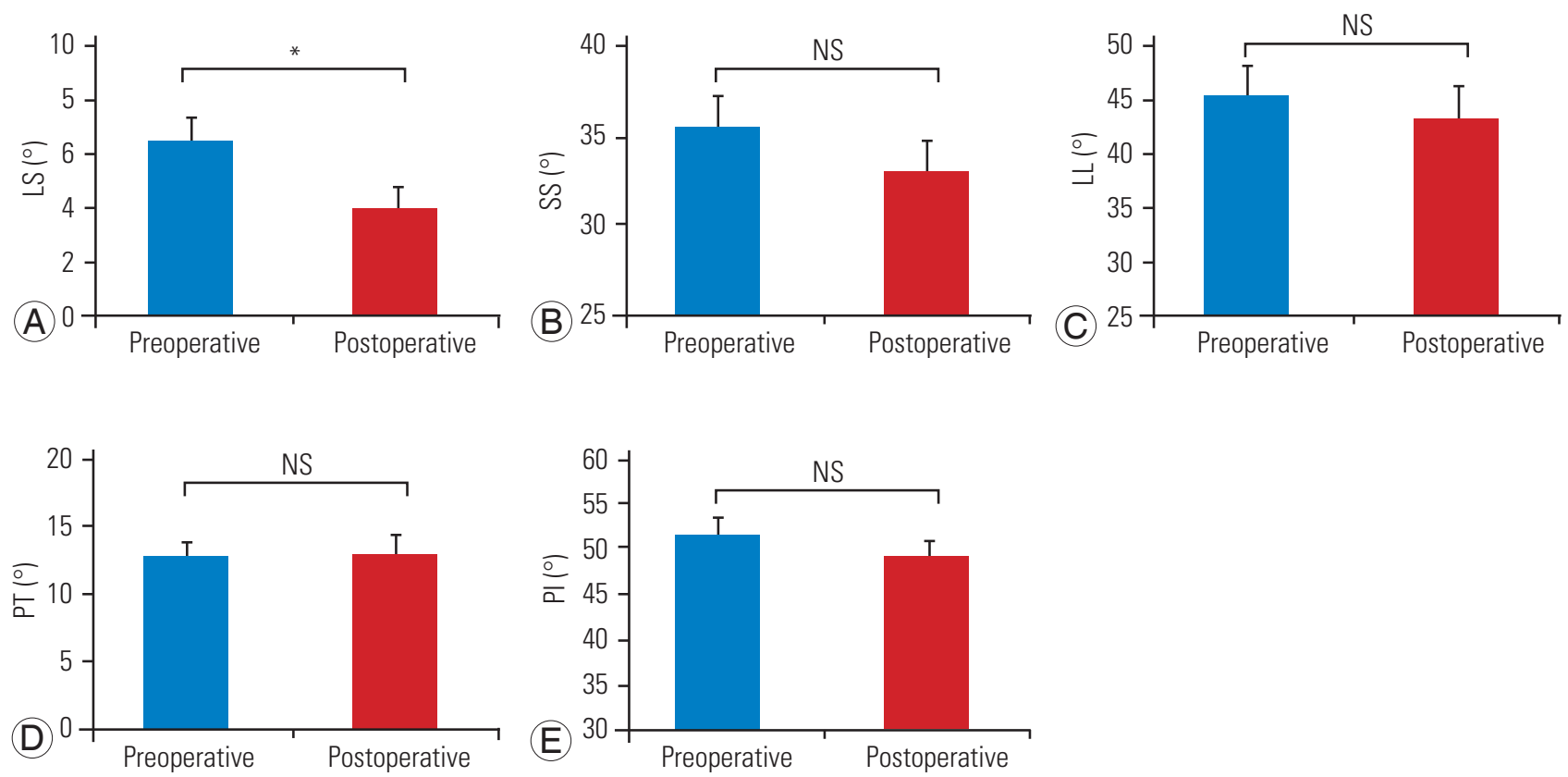

Fig. 4. Coronal and sagittal spinopelvic alignments of patients before and after total hip arthroplasty. Regarding spinal alignment metrics, (A) LS in the coronal alignment significantly reduced from preoperative $\left(6.557^{\circ}\right)$ to final observation $\left(3.969^{\circ}\right)(p=0.0194)$. On the other hand, there were no significant

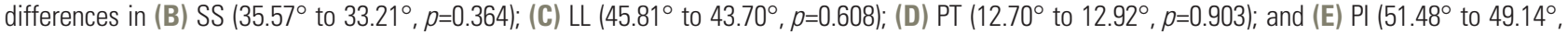
$p=0.378$ ) from preoperative-to-final observation. NS, not applicable; LS, lumbar scoliosis; SS, sacral slope; LL, lumbar lordosis; PT, pelvic tilt; PI, pelvic incidence. ${ }^{*} p<0.05$.

Table 3. The comparison of radiographic parameters and clinical symptoms between unilateral and bilateral hip osteoarthritis

\begin{tabular}{|c|c|c|c|c|c|c|}
\hline \multirow{2}{*}{ Variable } & \multicolumn{3}{|c|}{ Unilateral ( $n=18$, mean age $=64$ yr) } & \multicolumn{3}{|c|}{ Bilateral ( $n=12$, mean age $=62 \mathrm{yr}$ ) } \\
\hline & Preoperative & Final & $p$-value & Preoperative & Final & $p$-value \\
\hline \multicolumn{7}{|l|}{ Radiographic parameters } \\
\hline Lumbar scoliosis $\left({ }^{\circ}\right)$ & $7.21 \pm 1.14$ & $4.12 \pm 0.85$ & 0.037 & $5.50 \pm 1.16$ & $3.73 \pm 1.16$ & 0.29 \\
\hline Sacral slope $\left(^{\circ}\right)$ & $35.39 \pm 1.98$ & $33.38 \pm 1.81$ & 0.46 & $35.8 \pm 3.77$ & $32.96 \pm 3.60$ & 0.59 \\
\hline Lumbar lordosis $\left({ }^{\circ}\right)$ & $48.35 \pm 3.33$ & $45.89 \pm 3.35$ & 0.61 & $42.0 \pm 5.46$ & $40.42 \pm 5.03$ & 0.83 \\
\hline Pelvic tilt $\left({ }^{\circ}\right)$ & $11.36 \pm 1.25$ & $10.97 \pm 1.54$ & 0.84 & $14.78 \pm 2.28$ & $16.26 \pm 2.42$ & 0.66 \\
\hline Pelvic incidence $\left({ }^{\circ}\right)$ & $49.28 \pm 1.63$ & $47.89 \pm 1.90$ & 0.58 & $55.09 \pm 4.04$ & $51.4 \pm 3.96$ & 0.52 \\
\hline \multicolumn{7}{|l|}{ Clinical symptoms } \\
\hline VAS (low back pain) (mm) & $21.18 \pm 6.15$ & $4.76 \pm 1.93$ & 0.016 & $25.27 \pm 7.55$ & $8.10 \pm 4.08$ & 0.07 \\
\hline VAS (coxalgia) (mm) & $59.65 \pm 6.31$ & $2.12 \pm 0.75$ & 0.0000001 & $69.50 \pm 6.69$ & $6.64 \pm 6.34$ & 0.000001 \\
\hline Roland-Morris Disability Questionnaire & $4.72 \pm 1.07$ & $0.83 \pm 0.52$ & 0.002 & $6.17 \pm 1.90$ & $2.42 \pm 1.44$ & 0.13 \\
\hline
\end{tabular}

Values are presented as mean \pm standard error. Bold type is considered statistically significant $(p<0.05)$.

VAS, Visual Analogue Scale. 
from preoperative-to-final observation (Table 3).

A plain film of a typical case is shown in Figs. 6 and 7. Fig. 6 presents the image a 68-year-old female patient with unilateral right-sided $\mathrm{OA}$ who underwent a rightsided THA. Blood loss was $831 \mathrm{~mL}$, and operation time was 105 minutes.

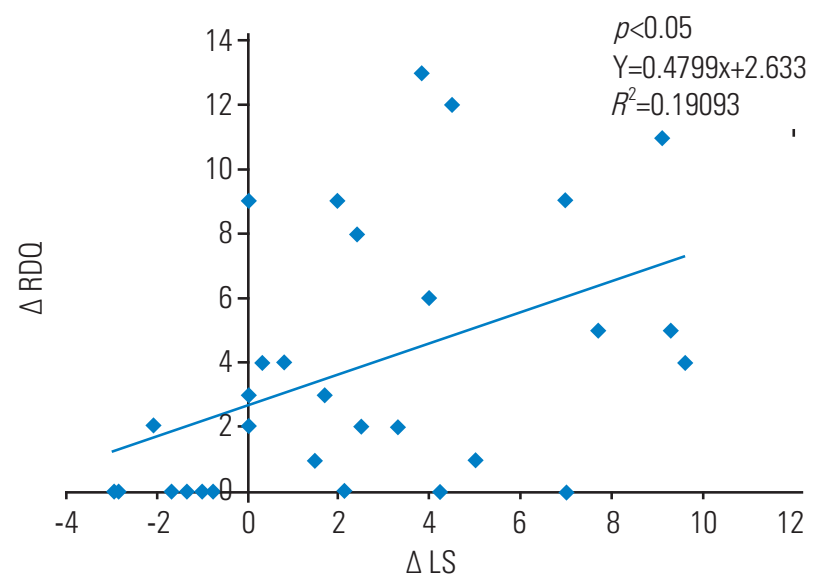

Fig. 5. Correlation between RDO and lumbar lordosis. RDO difference between the preoperative-to-postoperative observations correlated with the LS difference between the preoperative-to-postoperative observations ( $r=0.437, p=0.015)$. RDQ, Roland-Morris Disability Questionnaire; LS, lumbar scoliosis.
Preoperative, 1-month postoperative, and final LBP VAS scores markedly improved, progressing from $95 \mathrm{~mm}$ to $0 \mathrm{~mm}$ to $0 \mathrm{~mm}$, respectively; similarly, hip pain VAS score improved from $90 \mathrm{~mm}$ to $0 \mathrm{~mm}$ to $0 \mathrm{~mm}$, respectively, and preoperative-to-final RDQ improved from 12 to 0 , respectively. Regarding the spinal alignment metrics, preoperative-to-final LS improved from 8.4 to 3.9, respectively.

Fig. 7 presented the image of a 75-year-old female bilateral OA undergoing a right THA. Blood loss was $610 \mathrm{~mL}$ of blood loss, and operation time was 91 minutes.

Preoperative, 1-month postoperative, and final LBP VAS scores progressed from $25 \mathrm{~mm}$ to $43 \mathrm{~mm}$ to $43 \mathrm{~mm}$, respectively, whereas hip pain VAS scores improved markedly, progressing from $75 \mathrm{~mm}$ to $60 \mathrm{~mm}$ to $0 \mathrm{~mm}$, respectively. Preoperative-to-final RDQ remained at 0 throughout the follow-up period. In terms of spinal alignment, the preoperative-to-final LS progressed from 0.45 to 0.12 , respectively, which was not a significant change.

\section{Discussion}

Severe hip OA has been reported to cause spinal align-
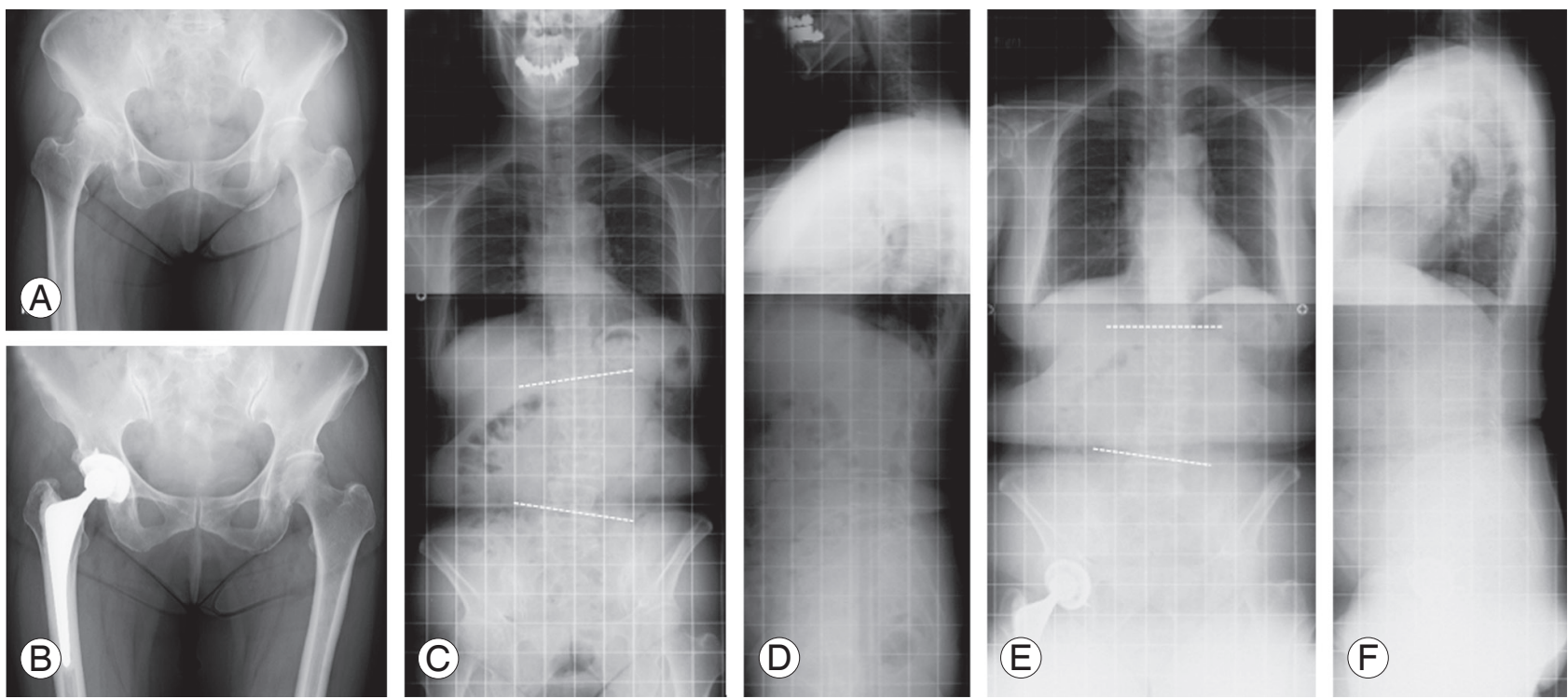

Fig. 6. Unilateral OA (68-year-old woman, right-sided OA). The patient underwent right-sided total hip arthroplasty. Blood loss was $831 \mathrm{~mL}$, and operation time was 105 minutes. (A) Preoperative hip joint plain film frontal view; (B) postoperative plain film frontal view; (C) preoperative whole spine plain film frontal view; (D) lateral view; and postoperative final total spinal column plain film (E) frontal view and (F) lateral view. Preoperative, 1-month postoperative, and final low back pain VAS scores were $95 \mathrm{~mm}, 0 \mathrm{~mm}$, and $0 \mathrm{~mm}$, respectively; hip pain VAS scores also markedly improved from $90 \mathrm{~mm}$ to $0 \mathrm{~mm}$ to $0 \mathrm{~mm}$, respectively. Roland-Morris Disability Questionnaires (preoperative, final) improved from 12 to 0 , respectively. The spinal alignment metrics were (preoperative to final, respectively, all) LS $\left(8.4^{\circ}\right.$ to $\left.3.9^{\circ}\right)$; sacral slope $\left(20^{\circ}\right.$ to $\left.23^{\circ}\right)$; lumbar lordosis $\left(36^{\circ}\right.$ to $\left.29^{\circ}\right)$; pelvic tilt $\left(22^{\circ}\right.$ to $\left.24^{\circ}\right)$; and pelvic incidence $\left(63^{\circ}\right.$ to $\left.64^{\circ}\right)$, with significant improvement in LS. OA, osteoarthritis; VAS, Visual Analogue Scale; LS, lumbar scoliosis. 

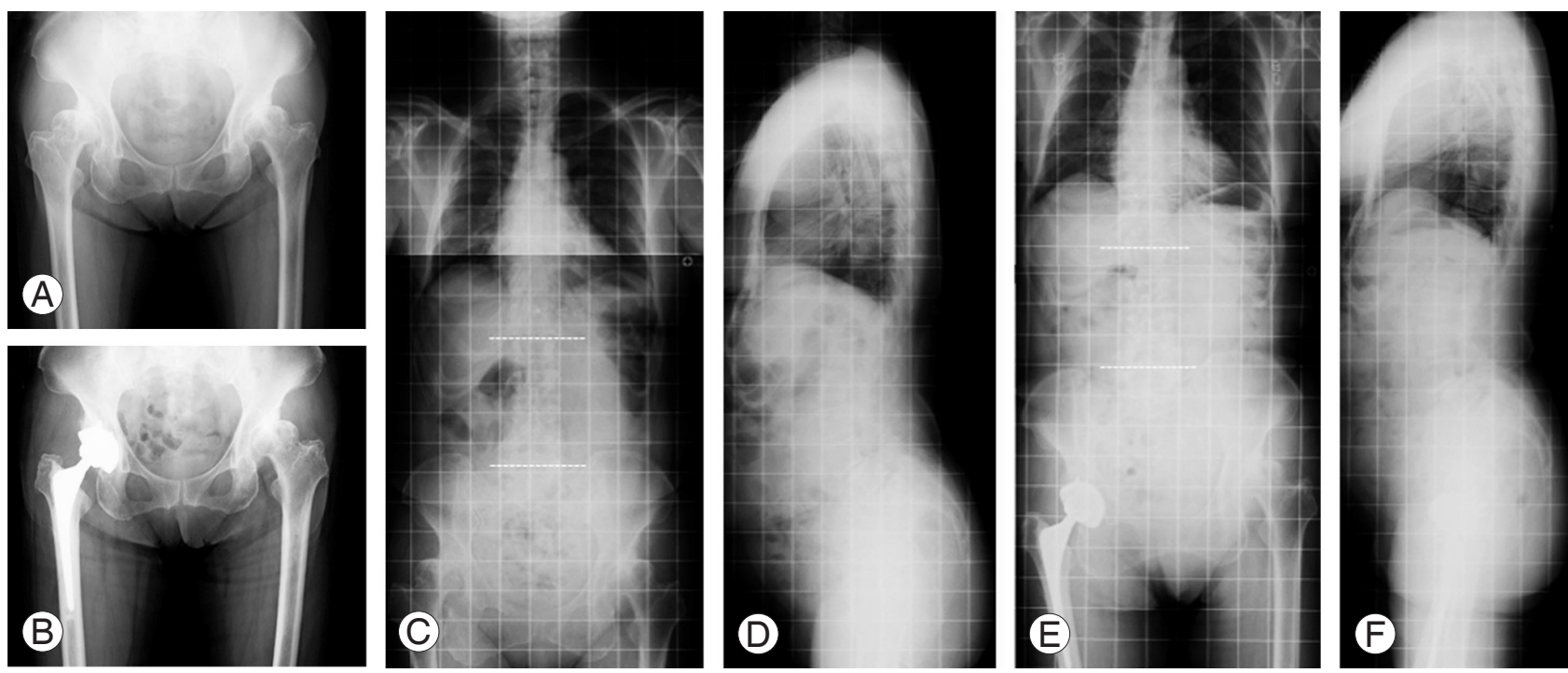

Fig. 7. Bilateral osteoarthritis (75-year-old woman). The patient underwent a right total hip arthroplasty. Blood loss was $610 \mathrm{~mL}$, and operation time was 91 minutes. (A) Preoperative hip joint plain film frontal view; (B) postoperative plain film frontal view; (C) preoperative whole spine plain film frontal view; (D) lateral view; postoperative final total spinal column plain film (E) frontal view and (F) lateral view. Preoperative, 1-month postoperative, and final low back pain VAS scores remained the same $(25 \mathrm{~mm}, 43 \mathrm{~mm}$, and $43 \mathrm{~mm}$, respectively), and hip pain VAS scores remarkably improved (from $75 \mathrm{~mm}$ to $60 \mathrm{~mm}$ to $0 \mathrm{~mm}$, respectively). Roland-Morris Disability Questionnaires (preoperative, final) remained at 0 . Spinal alignment metrics (preoperative to final, respectively, all) were lumbar scoliosis $\left(0.45^{\circ}\right.$ to $\left.0.12^{\circ}\right)$; sacral slope $\left(65^{\circ}\right.$ to $\left.55^{\circ}\right)$; lumbar lordosis $\left(71^{\circ}\right.$ to $\left.65^{\circ}\right)$; pelvic tilt $\left(11^{\circ}\right.$ to $\left.1^{\circ}\right)$; and pelvic incidence $\left(77^{\circ}\right.$ to $\left.70^{\circ}\right)$, with no significant changes. VAS, Visual Analogue Scale.

ment abnormalities and LBP, and LBP has been reported to improve following THA. Lumbosacral spinal alignment has been presumed to change following THA. Nevertheless, previous studies have revealed that spinal sagittal alignment did not differ following THA [2,7]. Additionally, there have been no reports regarding coronal alignment before and after THA.

Regarding spinal alignment, which adversely affects the quality of life (QOL), Takemitsu et al. [8] have reported that $95 \%$ of the patients with degenerative lumbar kyphosis complain of LBP with a severe disruption of ADL related to the kyphosis. Glassman et al. [9] have noted that patients with large sagittal vertical axes (SVA) in which the C7 plumb line shows anterior displacement suffer the greatest disruption of QOL and have stressed the importance of sagittal alignment. Lafage et al. [10] have reported an association of posterior PT and stooping posture with poor QOL and have considered PT and SVA as vital factors.

Regarding spinopelvic alignment related to hip OA, Okuda et al. [11] have reported that SS and LL angles were significantly greater in patients with hip OA than in healthy volunteers. Although in healthy subjects, aging is associated with the reduction in LL angle and posterior sacral inclination, the patients maintained LL and SS.

Yoshimoto et al. [12] have found that LL, slopes, and PI in the sagittal spinopelvic plane and pelvic obliquity and sharp angle in the coronal spinopelvic plane were significantly greater in patients with hip OA, suggesting that both sagittal alignment and coronal alignment metrics, such as pelvic obliquity, are associated with LBP in patients with hip OA.

In the present study, changes in coronal and sagittal spinopelvic alignments following THA were studied along with comprehensive changes in back pain, as measured by a scale for LBP with the patient in a standing position, JOABPEQ, and RDQ. LBP VAS scores, RDQ, and all JOABPEQ domains showed improvements following THA. Moreover, LS significantly reduced, and RDQ improvement was correlated with LS improvement. In analysis on the basis of laterality, improvements in LS and back pain were observed in patients with unilateral OA, whereas there were no significant improvements in spinal alignment and back pain in patients with bilateral OA.

Offierski and MacNab [1] has pointed out that the flexion contracture of the hip elicits an increased pelvic forward tilt and LL and that these induce LBP. In the present study, we did not detect pelvic forward tilt or lumbar 
hyperlordosis. Following surgery, the metrics of sagittal alignment, such as SS and LL, showed a downward trend, but there were no significant differences.

In hip OA, pelvic forward tilt generally occurs in young patients, whereas posterior tilt is common in the elderly $[11,13]$. In the present study including elderly patients, pelvic anteversion may have been offset by a secular pelvic posterior tilt and the progression of lumbar spine kyphosis, leading to an unchanged postoperative sagittal alignment.

Preoperative LS significantly reduced following THA. In such cases, PT due to leg length difference because of acetabular insufficiency caused a compensatory lumbar spine scoliosis.

Reportedly, leg length difference can be corrected and reversible compensatory scoliosis can be improved by restoring the normal acetabular structure following THA. Furthermore, the correlation between RDQ score improvement and LS improvement following THA suggests that hip OA compensatory LS may be involved in LBP.

In patients with bilateral OA, diminished hip flexion and LS did not appear to improve and back pain indeed remained after THA because of hip joint dysfunction and a further decline in the contralateral side. On the basis of these results, we believe that improvements in compensatory LS may be involved in improvements in back pain following THA.

Our study has several limitations. First, only a small number of subjects were investigated, which requires the confirmation of our findings in a larger population. Second, our study comprised 6 months of follow-up after THA, yet there is an additional long-term follow-up of LBP and spinal alignment changes should be conducted. Third, our study suggests that sagittal alignment may not change in the elderly; however, changes in sagittal spinopelvic alignment before and after THA should be compared across various age groups, including juvenile patients. Fourth, we did not conduct comprehensive measurements of whole spine alignment, including global balance parameters such as SVA. Finally, we did not measure leg length differences before and after surgery. These limitations should be considered when interpreting our results and should be addressed in the future studies.

\section{Conclusions}

We evaluated effects of THA on spinopelvic alignment and LBP in patients with severe hip OA. Both LBP and spinal alignment metrics, such as LS, improved after THA. Improvement in LS and back pain were observed in patients with unilateral OA; however, there were no significant improvements in spinal alignment or back pain metrics in patients with bilateral OA. LS and RDQ were strongly correlated, indicating that abnormal coronal lumbosacral alignment, such as LS, affects LBP.

\section{Conflict of Interest}

No potential conflict of interest relevant to this article was reported.

\section{References}

1. Offierski CM, MacNab I. Hip-spine syndrome. Spine (Phila Pa 1976) 1983;8:316-21.

2. Ben-Galim P, Ben-Galim T, Rand N, et al. Hip-spine syndrome: the effect of total hip replacement surgery on low back pain in severe osteoarthritis of the hip. Spine (Phila Pa 1976) 2007;32:2099-102.

3. Parvizi J, Pour AE, Hillibrand A, Goldberg G, Sharkey PF, Rothman RH. Back pain and total hip arthroplasty: a prospective natural history study. Clin Orthop Relat Res 2010;468:1325-30.

4. Fukui M, Chiba K, Kawakami M, et al. JOA Back Pain Evaluation Questionnaire (JOABPEQ)/JOA Cervical Myelopathy Evaluation Questionnaire (JOACMEQ): the report on the development of revised versions; April 16, 2007; The Subcommittee of the Clinical Outcome Committee of the Japanese Orthopaedic Association on Low Back Pain and Cervical Myelopathy Evaluation. J Orthop Sci 2009;14:34865.

5. Roland M, Morris R. A study of the natural history of back pain: part I: development of a reliable and sensitive measure of disability in low-back pain. Spine (Phila Pa 1976) 1983;8:141-4.

6. O’Brien MF, Kuklo TR, Blanke KM, Lenke LG; Spinal Deformity Study Group. Radiographic measurement manual. Memphis (TN): Medtronic Sofamor Danek USA; 2008.

7. Radcliff KE, Orozco F, Molby N, et al. Change in spinal alignment after total hip arthroplasty. Orthop Surg 2013;5:261-5. 
8. Takemitsu Y, Harada Y, Iwahara T, Miyamoto M, Miyatake Y. Lumbar degenerative kyphosis: clinical, radiological and epidemiological studies. Spine (Phila Pa 1976) 1988;13:1317-26.

9. Glassman SD, Bridwell K, Dimar JR, Horton W, Berven S, Schwab F. The impact of positive sagittal balance in adult spinal deformity. Spine (Phila Pa 1976) 2005;30:2024-9.

10. Lafage V, Schwab F, Patel A, Hawkinson N, Farcy JP. Pelvic tilt and truncal inclination: two key radiographic parameters in the setting of adults with spinal deformity. Spine (Phila Pa 1976) 2009;34:E599606.
11. Okuda T, Fujita T, Kaneuji A, Miaki K, Yasuda Y, Matsumoto T. Stage-specific sagittal spinopelvic alignment changes in osteoarthritis of the hip secondary to developmental hip dysplasia. Spine (Phila Pa 1976) 2007;32:E816-9.

12. Yoshimoto H, Sato S, Masuda T, et al. Spinopelvic alignment in patients with osteoarthrosis of the hip: a radiographic comparison to patients with low back pain. Spine (Phila Pa 1976) 2005;30:1650-7.

13. Matsuyama Y, Hasegawa Y, Yoshihara H, et al. Hipspine syndrome: total sagittal alignment of the spine and clinical symptoms in patients with bilateral congenital hip dislocation. Spine (Phila Pa 1976) 2004;29:2432-7. 